Kev. Saúde públ., S. Paulo, 19:450-7, 1985.

\title{
A ANEMIA NUTRICIONAL ENTRE GESTANTES ATENDIDAS EM CENTROS DE SAUUDE DO ESTADO DE SÃO PAULO (BRASIL)*
}

\author{
Sophia Cornbluth Szarfarc**
}

SZARFARC, S.C. A anemia nutricional entre gestantes atendidas em centros de saúde do Estado de São Paulo (Brasil). Rev. Saúde públ., S. Paulo, 19:450-7, 1985.

RESUMO: Com o objetivo de estimar a prevalência de anemia entre a clientela do Programa de Atendimento à Gestante (PAG), da Secretaria de Saúde do Estado de São Paulo (Brasil), e de analisar o item referente à suplementação de ferro, do mesmo programa, estudaram-se os prontuários de 4.539 gestantes, matriculadas nos PAGs de 15 Centros de Saúde (CS), escolhidos através de amostragem, probabilística. Observou-se anemia (hemoglobina - $\mathrm{Hb}-<11,0 \mathrm{~g} / \mathrm{dl}$ ) em 35,1\% das gestantes, valor que sugere constituir essa deficiência problema de saúde pública em relação à clientela dos Centros de Saúde estudados. E destacada a necessidade de incluir, na rotina do PAG, a medida da concentração de $\mathrm{Hb}$ e de referi-la conjuntamente com a idade gestacional propiciando conhecer a necessidade de ferro suplementar específica de cada grávida, aumentando, com isso, a eficiência do programa de combate à anemia e, conseqüentemente, a do PAG. Sugere-se também que a avaliação da suplementação seja feita em Centros de Saúde que tenham facilidade para tal.

UNITERMOS: Anemia ferropriva, prevenção e controle. Gestantes, estado nutricicnal. Ferro, uso terapêtico. Programa de Atendimento à Gestante (PAG).

\section{INTRODUÇÃO}

Tendo em vista que o grupo gestante é o mais sensível à deficiência de ferro $\mathrm{e}$ às suas consequiências, e considerando que a ingestão de ferro suplementar é tida como a forma mais factível de combate a essa deficiência nutricional, instituições como a Organização Mundial da Saúde (OMS) ${ }^{\text {1n }}$ e "International Nutritional Anemia Consultative Group" (INACG) ${ }^{6}$ recomendam a inclusão compulsória deste suplemento nos programas de pré-natal, mesmo em áreas de baixa prevalência de anemia.

Nos serviços públicos de pré-natal do Estado de São Paulo, parcelas elevadas de mulheres aliam à situação fisiológica de demanda aumentada de ferro, a situação sócio-econômica precária, o que facilita a ocorrência de anemia (dieta ina- dequada, infestações parasitárias) e torna esse grupo de maior risco que os demais.

A demanda por serviços institucionais de assistência pré-natal, entre nós, é elevada. Baseado nos Censos de $1970^{4} \mathrm{e}$ de $1980^{5}$ e em informações do CIS * (Centro de Informações da Saúde), estima-se que cerca de $20 \%$ das gestantes paulistas estão matriculadas no Programa de Atendimento à Gestante (PAG) da Secretaria de Saúde de São Paulo.

Estas considerações justificam e ressaltam a importância da indicação de suplementação de ferro, feita à gestante matriculada no PAG ${ }^{1.1}$ de rotina.

Chama a atenção não haver nesse Programa qualquer referência à avaliação quer na fase de identificação da

\footnotetext{
- Trabalho realizado com auxílio financeiro do Instituto Nacional de Alimentação e Nutrição -- INAN Convênio $08 / 81$.

* Do Departamento de Nutrição da Facuidade de Saúde Pública da Universidade de São Paulo Av. Dr. Arnaldo. 715 - 01255 - São Paulo. SP - Brasil

\#* Centro pertencente à Secretaria da Saúde do Estado de São Paulo - Dados inéditos.
} 
SZARFARC, S.C. A anemia nutricional entre gestantes atendidas em centros de saúde do Estado de São Paulo (Brasil). Rev. Saúde públ., S. Paulo, 19:450-7, 1985.

ocorrência da anemia, quer com relação a eficiência, eficácia e efetividade do suplemento no combate a essa carência nutricional.

Este trabalho foi realizado com o objetivo de avaliar a prevalência de anemia entre a clientela do PAG e discutir, com base nos valores encontrados, a validade da suplementação, na forma em que ela é realizada.

\section{METODOLOGIA}

Fixou-se para estudo o período de tempo compreendido entre setembro de 1977 a dezembro de 1981.

Preliminarmente, selecionou-se os Centros de Saúde (CS) do Estado de São Paulo que solicitaram, do Instituto Adolfo Lutz (IAL), 360 ou mais "análises clínicas" para gestantes *, no $4 .^{\circ}$ trimestre do ano de 1981. Este valor mínimo teve como objetivo garantir um número ponderoso de gestantes na amostra.

Os $57 \mathrm{CS}$ que atenderam a essa condição foram agrupados segundo a Divisão Regional de Saúde (DRS) a que pertencem, constituindo os estratos de onde foi retirada a amostra (que foi de tamanha proporcional ao número de $\mathrm{CS}$ constantes em cada DRS), com número total de unidades igual a $39(68 \%)$.

Através da amostragem casual sistemática e/ou do agendamento laboratorial, identificaram-se os prontuários das gestantes.

$\mathrm{Na}$ maior parte dos CS amostrados, a solicitação da medida da concentração de $\mathrm{Hb}$ somente é feita quando há algu-

\footnotetext{
* A totalidade dos exames laboratoriais dos CS são realizados nos laboratórios do IAL. No Boletim Trimestral de Procedência de Amostras do IAL (boletim de circulação interna), sob o nome de "análises clínicas", estão agrupados os exames laboratoriais, inclusive aqueles que identificam a anemia: hemoglobina, hemograma, etc., distribuídos segundo grupos populacionais entre os quais aquele de nosso interesse: o das gestantes.
}

ma evidência clínica da presença de anemia. Assim, a fim de eliminar a tendenciosidade dos resultados, decidiu-se excluir da amostra os CS que não tivessem realizado, como rotina no $P A G$, no período estudado, a medida da concentração de hemoglobina pelo método colorimétrico da cianometahemoglobina. Foram 24 os CS excluídos, permanecendo $15(38,5 \%)$ com 4.539 unidades. Por ter havido uma seleção inicial na escolha dos CS e outra determinada pela rotina do PAG, não podemos afirmar que os resultados sejam representativos de todos os CS.

Das diversas informações colhidas, vamos nos ater às referentes à concentração de hemoglobina $(\mathrm{Hb})$ e idade gestacional (IG) por ocasião da medida do indicador bioquímico. O diagnóstico de anemia foi feito segundo os valores críticos sugeridos pela OMS ${ }^{7}$ e Szarfarc e col. ${ }^{14}$.

\section{RESULTADOS E COMENTÁRIOS}

A anemia, definida pelo parâmetro proposto pela OMS $^{8}$ - concentração de $\mathrm{Hb}$ inferior a $11,0 \mathrm{~g} / \mathrm{dl}$ - atingiu a $35,1 \%$ da população amostral. (Tabela 1).

A Comissão de Estudos para Anemias Nutricionais, reunida pelo Instituto Nacional de Alimentação e Nutrição (INAN), em julho de 1982, com o objetivo de formular estratégias de intervenção no combate à anemia ferropriva, destaca a gestante como "Grupo Vulnerável Indicador" - GVI * e o valor $11,0 \mathrm{~g} / \mathrm{dl}$ para $\mathrm{Hb}$ como crítico na caracterização de anemia nesse grupo. **

\footnotetext{
* GVI - Expressão de autoria de Yaro Ribeiro Gandra. A partir da medida de prevalência de uma desnutrição no GVI que é, habitualmente, o grupo mais sensível a essa patologia, é possível detectar o risco de ocorrência da deficiência em outros grupos etários e/ou fisiológicos.

** Informaçōes não publicadas.
} 
SZARFARC, S.C. A anemia nutricional entre gestantes atendidas em centros de saúde do Estado de São Paulo (Brasil). Rev. Saúde públ., S. Paulo, 19:450-7, 1985.

TABELA 1

Prevalência de anemia $(\mathrm{Hb}<11,0 \mathrm{~g} / \mathrm{dl})$ em gestantes matriculadas nos PAG dos CS do Estado de São Paulo, setembro 1977/dezembro 1981.

\begin{tabular}{|c|c|c|c|c|}
\hline \multirow{2}{*}{$\mathrm{CS}$} & \multirow{2}{*}{ DRS } & \multirow{2}{*}{$\frac{\text { Tamanho da amostra }}{\text { N. }{ }^{\circ}}$} & \multicolumn{2}{|c|}{ Gestantes anêmicas } \\
\hline & & & N. ${ }^{\circ}$ & $\%$ \\
\hline Lapa & 1 & 257 & 20 & 7,8 \\
\hline São Miguel Paulista & 1 & 252 & 22 & 8,7 \\
\hline Mauá & 1 & 386 & 158 & 40,9 \\
\hline São José dos Campos & 3 & 356 & 172 & 48,3 \\
\hline Jacareí & 3 & 611 & 246 & 40,6 \\
\hline Sorocaba & 4 & 261 & 134 & 51,3 \\
\hline Campinas & 5 & 402 & 321 & 65,2 \\
\hline Casa Branca & 5 & 260 & 23 & 8,8 \\
\hline Arthur Nogueira & 5 & 431 & 198 & 45,9 \\
\hline Ribeirão Preto & 6 & 189 & 54 & 28,6 \\
\hline São Carlos & 6 & 195 & 19 & 9,7 \\
\hline Baurư & 7 & 254 & 16 & 6,3 \\
\hline São José do Rio Preto & 8 & 249 & 87 & 34,9 \\
\hline Araçatuba & 9 & 250 & 99 & 39,6 \\
\hline Marília & 11 & 96 & 24 & 25,0 \\
\hline Total & & 4.539 & 1.593 & 35,1 \\
\hline
\end{tabular}

CS: Centros de Saúde da Secretaria da Saúde do Estado de São Paulo (SS).

DRS: Divisão Regional de Saúde da SS.

Com base nessas premissas propôs o esquema abaixo para quantificar o risço de anemia na população geral.

\begin{tabular}{cc}
\hline Níveis de risco & $\begin{array}{c}\text { \% de gestantes com } \\
\mathrm{Hb}<11,0 \mathrm{~g} / \mathrm{dl}\end{array}$ \\
\hline I & $<10$ \\
II & $\geqslant 10<30$ \\
III & $\geqslant 30$ \\
\hline
\end{tabular}

A Tabela 1 indica diferentes níveis de risco para as diversas áreas estudadas, mostrando que há grande variação de prevalência de anemia nas diferentes unidades sanitárias e apontando para a necessidade imperiosa de quantificar a anemia e, com isso, capacitar os serviços de saúde a discernir sobre as necessidades específicas de cada grupo populacional, no que se refere à deficiência de ferro. Para situações de níveis de risco elevado, como ocorre em $53 \%$ das comunidades estudadas, a OMS ${ }^{10}$ e o INACG ${ }^{6}$ recomendam programas de suplementação para a totalidade da população, visando com isso controlar a anemia nutricional - melhorar, em consequiência, as condições de desempenho e a qualidade de vida da mesma.

A quantificação da concentração de hemoglobina no diagnóstico de deficiência de ferro é de grande importância no pré-natal. Este indicador bioquímico reflete, de alguma forma, a qualidade de vida (tendo em vista as causas principais na gênese da anemia) e é freqüuentemente 
SZARFARC, S.C. A anemia nutricional entre gestantes atendidas em centros de saúde do Estado de São Paulo (Brasil). Rev. Saúde públ., S. Paulo, 19:450-7, 1985.

relacionado a eventos mórbidos da gestação. Por isso, constitui um fator de risco reprodutivo 2,9 , podendo ser utilizado como indicador do mesmo ${ }^{13}$.

A medida da concentração de $\mathrm{Hb}$ foi realizada por ocasião da inscrição das grávidas ao PAG, juntamente com os exames laboratoriais de rotina ( $\mathrm{Hb}$, tipagem, VDRL) em todos os CS amostrados. Sendo a idade gestacional fator de influência na concentração de $\mathrm{Hb}$, é de interesse conhecer os valores deste indicador quando relacionados à IG (Tabela 2).

\section{TABELA 2}

$\mathrm{Hb}$ (média e desvio-padrão) segundo idade gestacional em CS do Estado de São Paulo, setembro 1977 /dezembro 1981.

\begin{tabular}{cccc}
\hline $\begin{array}{c}\text { Idade gestacional } \\
\text { (meses) }\end{array}$ & Tamanho da amostra & \multicolumn{2}{c}{$\frac{\text { [Hb] }}{\mathrm{X}} \mathrm{g} / \mathrm{d} \mathrm{s}$} \\
\hline 1 & 122 & 12,07 & 1,56 \\
2 & 656 & 12,00 & 1,56 \\
3 & 773 & 11,87 & 1,43 \\
4 & 848 & 11,54 & 1,44 \\
5 & 681 & 11,27 & 1,43 \\
6 & 520 & 11,23 & 1,33 \\
7 & 378 & 11,21 & 1,38 \\
8 & 187 & 11,00 & 1,52 \\
9 & 043 & 11,25 & 1,55 \\
\hline Total & $4.208 *$ & 11,55 & 1,47 \\
\hline
\end{tabular}

* 331 gestantes não tinham registrado a sua idade gestacional.

Nota-se nesta Tabela a tendência, já descrita em outros trabalhos ${ }^{3,7,14}$, de manutenção dos valores de $\mathrm{Hb}$ no início da gestação, seguida de sua diminuição e de ligeiro aumento no final do processo.

O valor proposto pela OMS ${ }^{8}$ para diagnosticar anemia, por ser constante para todo período de gravidez, não se adapta a esse modelo de variação de $\mathrm{Hb}$. Resulta disso variação nos níveis de sensibilidade e especificidade do indicador quando utilizado em mulheres com diferentes IG.

Uma forma que tem sido adotada para manter constante a especificidade $e$ sensibilidade é a escolha de valores de uma curva de referência correspondente a um determinado percentil ${ }^{1}$.

Estimamos (Tabela 3) o risco de ane- mia entre a clientela do PAG utilizando dois parâmetros, respectivamente sem e com níveis de especificidade/sensibilidade constantes:

1) - padrão OMS ${ }^{8}$.

2) - valores de $\mathrm{Hb}$ correspondentes, em cada idade gestacional, ao percentil $7(\overline{\mathrm{X}}-1,5 \mathrm{~s}) *$ da população de referência ${ }^{14}$.

Como mostram os valores da Tabela 3, a proporção de mulheres anêmicas é expressivamente diferente nas diferentes IG, na dependência do valor crítico para diagnóstico.

O valor único, proposto pela OMS, além de não assimilar o modelo segundo o qual a concentração de $\mathrm{Hb}$ varia,

\footnotetext{
* Baseado em Viteri e col.15
} 
SZARFARC, S.C. A anemia nutricional entre gestantes atendidas em centros de saúde do Estado de São Paulo (Brasil). Rev. Saúde públ., S. Paulo, 19:450-7, 1985.

\section{TABELA 3}

Estimativa*, por idade gestacional, da percentagem de mulheres anêmicas, em $\mathrm{CS}$ do Estado de São Paulo, segundo dois índices.

\begin{tabular}{|c|c|c|c|c|}
\hline \multirow{2}{*}{$\begin{array}{c}1 \mathrm{G} \\
\text { (Meses) }\end{array}$} & \multicolumn{2}{|l|}{ OMS ${ }^{8}$} & \multicolumn{2}{|c|}{ Szarfarc e col. ${ }^{12}$} \\
\hline & $\begin{array}{l}\text { Valor de referência } \\
\mathrm{gHb} / \mathrm{dl}\end{array}$ & $\begin{array}{c}\text { Anemia } \\
\%\end{array}$ & $\begin{array}{l}\text { Valor de referência } \\
\qquad \mathrm{gHb} / \mathrm{dl}\end{array}$ & $\begin{array}{l}\text { Anemia } \\
\%\end{array}$ \\
\hline 1 & 11,00 & 27 & 12,00 & 49 \\
\hline 2 & 11,00 & 28 & 12,00 & 49 \\
\hline 3 & 11,00 & 29 & 12,00 & 56 \\
\hline 4 & 11,00 & 37 & 11,75 & 59 \\
\hline 5 & 11,00 & 45 & 11,40 & 53 \\
\hline 6 & 11,00 & 45 & 11,35 & 55 \\
\hline 7 & 11,00 & 46 & 11,35 & 55 \\
\hline 8 & 11,00 & 50 & 11,30 & 57 \\
\hline 9 & 11,00 & 44 & 11,70 & 61 \\
\hline
\end{tabular}

* As estimativas de risco 12 foram obtidas a partir de valores padronizados $(\mathrm{X}-\overline{\mathrm{X}}) / \mathrm{s}$, onde $\mathrm{X}=$ valor de referência e $\overline{\mathrm{X}}=$ valores citados na Tabela 2 .

por ser fixo, não consegue (especialmente no início da gravidez, quando a necessidade de ferro extra-basal é insignificante), grande parte das vezes, identificar a anemia, confundindo-a com a depleção fisiológica própria do processo gravídico. O uso de valores obtidos de uma curva de referência permite diminuir sensivelmente este defeito.

Evidentemente, a escolha de um valor crítico de $\mathrm{Hb}$ depende, fundamentalmente, do objetivo ao qual ele se destina. O padrão OMS é, por exemplo,
- mais indicado para quantificar a prevalência de anemia em estudos comparativos. Por sua vez, a inexistência de uma curva de referência universalmente aceita (como é o padrão OMS) que refira valores de $\mathrm{Hb}$, segundo IG, limita o seu valor. Sua utilização, no entanto, em serviços de saúde, apesar desta restrição, é de grande valia na determinação do estado nutricional da grávida.

A necessidade extra de ferro de uma gestante pode ser esquematizada conforme pode ser verificado na Tabela 4 .

TABELA 4

Esquema da necessidade de ferro da gestante, por idade gestacional

\begin{tabular}{lrrrrrrrrrrr}
\hline & \multicolumn{1}{c}{ Idade gestacional (meses) } \\
\cline { 2 - 11 } Fe mg/mês & 1 & 2 & 3 & 4 & 5 & 6 & 7 & 8 & 9 & Total \\
\hline Expansão da volemia (1,21) & 04 & 12 & 32 & 39 & 61 & 95 & 114 & 101 & - & 458 \\
Feto + placenta & 06 & 11 & 15 & 30 & 38 & 47 & 65 & 93 & 63 & 370 \\
Perdas no parto & - & - & - & - & - & - & - & - & 150 & 150 \\
\hline Total & 10 & 23 & 47 & 69 & 99 & 142 & 182 & 194 & 213 & 1.178 \\
\hline
\end{tabular}

Fonte: Adaptado do Iron deficiency in Women 7 . 
SZARFARC, S.C. A anemia nutricional entre gestantes atendidas em centros de saúde do Estado de São Paulo (Brasil). Rev. Saúde públ., S. Paulo, 19:450-7, 1985.

Vale a pena analisar esses valores calculados, em conjunto com o sub-programa de suplementação de ferro apresentado ao PAG:

"Suplementação férrica: será indicado o suplemento de $60 \mathrm{mg}$ de Fe elementar por dia de rotina às gestantes a partir do $2 .^{\circ}$ trimestre de gestação, por um período mínimo de dois meses... Considerando-se que a absorção média do ferro ingerido é de $10 \%$, ao final de 60 dias teríamos a absorção mínima de $360 \mathrm{mg}$ de ferro elementar, o que equivale ao mínimo de ferro necessário para o feto. Dessa maneira, espera-se que não haja falta de ferro para a mãe e, ao mesmo tempo, como o percentual de absorção intestinal depende das necessidades reais, o oferecimento desta quantidade diária possibilitaria a correção de possíveis deficiências de base existentes". ${ }^{11}$

De acordo com o referido no PAG, a gestante poderá absorver até $1080 \mathrm{mg}$ de ferro do suplemento $(6 \mathrm{mg} / \mathrm{dia} \times 180$ dias), o que atenderá o total da demanda marcial imposta pela gestação. No entanto, a gestante que chega anêmica ao serviço de pré-natal ( $35 \%$ da população - Tabela 1) ou que se matricula com IG superior a 4 meses (quase $50 \%$ da clientela - Tabela 2) não terá corrigida a "deficiência de base existente" como se propõe o PAG a realizar.

Assim, quanto à suplementação de ferro, dois aspectos merecem ser destacados: o primeiro diz respeito ao estado nutricional de ferro da gestante e o segundo ao tempo disponível para que a parturiente atinja um adequado estado nutricional com relação ao mineral. Desta forma, o conhecimento da concentração de hemoglobina/idade gestacional é fundamental para que os aspectos acima mencionados possam ser considerados no atendimento à gestante.

Há ainda a acrescentar que, embora a quantidade de ferro ideal a ser oferecida à grávida possa ser calculada teoricamente com facilidade, a partir do valor de $\mathrm{Hb}$ e de IG, na prática, esse método pode ser ineficaz. E sobejamente conhecido (e mesmo citado no PAG) que a absorção do ferro dietético ou suplementar é regulada pelo aumento da requisição. Os valores $10 \%$ (referido pela Secretaria da Saúde) ${ }^{11}$ e $5 \%$ (referido pelo INACG) ${ }^{6}$ são obtidos em condições orgânicas padronizadas que, evidentemente, não são as encontradas entre as grávidas.

Somente através da avaliação sistemática da suplementação férrica poder-se-á estabelecer os valores ótimos de ferro a serem oferecidos para as gestantes. Por isso, para estabelecimento desses valores sugere-se que seja introduzida na rotina de laboratório do PAG a dosagem, de $\mathrm{Hb}$ e que, paralelamente, em $\mathrm{CS}$ que tenham facilidade de obtenção dos serviços laboratoriais do IAL, a dosagem de $\mathrm{Hb}$ seja repetida mensalmente.

E necessário enfatizar, ainda uma vez, que é o conhecimento da concentração de $\mathrm{Hb}$, feita por ocasião da matrícula ao PAG, que servirá de base para a oferta do nutriente em pauta e, também, que é o mesmo indicador $\mathrm{Hb}$ que permitirá avaliar a qualidade da conduta adotada propiciando condições para o aumento da eficiência do PAG.

\section{CONCLUSÕES}

A partir do conhecimento da concentração de hemoglobina e idade gestacional da clientela de 15 Centros de Saúde do Estado de São Paulo, concluiu-se:

- a anemia nutricional, identificada pela concentração de hemoglobina inferior a $11,0 \mathrm{~g} / \mathrm{dl}$, ocorre em $35,1 \%$ da população estudada, constituindo um problema de saúde pública para a população pertencente à área de atuação dos Centros de Saúde;

- a prevalência com que essa deficiência ocorre nas diversas unidades sanitárias estudadas é heterogênea indican- 
SZARFARC, S.C. A anemia nutricional entre gestantes atendidas em centros de saúde do Estado de São Paulo (Brasil). Rev. Saúde ptíbl., S. Paulo, 19:450-7, 1985.

do a necessidade de condutas diferentes nas diferentes regiões, no seu combate;

- destaca-se a necessidade de incluir na rotina do PAG a medida da concentração de $\mathrm{Hb}$;

- ressalta-se a necessidade de referir conjuntamente o valor da concentração de $\mathrm{Hb}$ e idade gestacional, dada a influência que a segunda exerce sobre a primeira;

- a ausência de valores padrão de $\mathrm{Hb}$ que considerem a variação desse compo- nente hematológico durante o decorrer do processo gravídico e que sejam indiscutivelmente aceitos, prejudica seu uso na estimativa de risco de deficiência de ferro entre grávidas;

- sugere-se a introdução da medida de concentração de $\mathrm{Hb}$ na rotina do PAG; em CS que tenham facilidade de obtenção de serviços laboratoriais do IAL, sugere-se que a medida da concentração de $\mathrm{Hb}$ seja repetida mensalmente permitindo a avaliação sistemática da suplementação férrica.

SZARFARC, S.C. [Nutritional anaemia in pregnant women attending health centers in S. Paulo State (Brazil)]. Rev. Saúđe públ,, S. Paulo, 19:450-7, 1985.

ABSTRACT: In order to estimate the prevalence of anaemia among pregnant women receiving attention at the "Program of Attention to Pregnant Women" (PAG) of the Secretariat of Health of the State of S. Paulo, and also with a view to analysing the item of PAG referring to iron supplementation, a probabilist sample of 4539 . drawn from 15 Health Centers (HC), was studied. Anaemia was observed in $35.1 \%$ of the women $(\mathrm{Hb}<11.0 \mathrm{~g} / \mathrm{dl})$, suggesting that iron deficiency is a considerable public health problem for the sample population. Based on these results the introduction of $\mathrm{Hb}$ determination as part of the "PAG" routine is suggested. Results should be related to gestational age. It is also suggested that, in some $\mathrm{HC}$, efficiency of iron supplementation be evaluated.

UNITERMS: Anaemia, hypochromic, prevention and control. Pregnancy, nutritional status. Iron therapeutic use.

\section{REFERENCIAS BIBLIOGRÁFICAS}

1. DALlman, P.R. \& SIIMES, M.A. Percentiles curves for hemoglobin and red cell volume in infancy and childhood J. Pediat., 94: 26-31, 1979.

2. DELASCIO, D. \& ALMEIDA, P.A.M. Propedêutica da gestaçāo de alto risco. São Paulo, Livraria Manole, 1974.

3. HYTTEN, F. \& LEITCH, I. The physiology of human pregnancy. London. Blackwell, 1964.

4. FUNDAÇĀO IBGE. Censo demográfico: São Paulo. Rio de Janeiro, 1970. (8. Recenseameno Geral. Série Regional. 1970, v. 1).

5. FUNDAÇÃO IBGE. Sinopse preliminar do censo demográfico: Brasil. Rio de Janeiro, 1981. (9.0 Recenseamento Ge. ral do Brasil, 1980, v, 1).
6. INTERNATIONAL NUTRITIONAL ANEMIA CONSULTATIVE GROUP (INACG). Guidelines for eradication of iron deficiency. New York, 1977.

7. INTERNATIONAL NUTRITIONAL ANEMIA CONSULTATIVE GROUP (INACG), Iron deficiency in women. New York, 1981.

8. ORGANIZAÇÃO MUNDIAL DA SAÚDE. Grupo Científico de Anemias Nutricionales, Ginebra, 1968. Informe. Ginebra, 1968. (Ser. Inf. técn., 457).

9. ORGANIZAÇÃO MUNDIAL DA SAÜDE. Risk approach for maternal and child health care. Geneva, 1978. (WHO Offset Publ., 39). 
SZARFARC, S.C. A anemia nutricional entre gestantes atendidas em centros de saúde do Estado de São Paulo (Brasil). Rev. Saúde públ., S. Paulo, 19:450-7, 1985.

10. REUNION MIXTA ADI/OIEA/OMS, Ginebra, 1970. Lucha contra la anemia nutricional, especialmente contra la carência de hierro. Ginebra, Organización Mundial de la Salud. 1975 (OMS Ser. Inf. tecn., 580).

11. SECRETARIA DE ESTADO DA SAÜDE. Coordenadoria da Comunidade. Programa de assistência à gestante. São Paulo, s.d. [Mimeografado].

12. SPIEGEL, M.R. Estatística. São Pæulo, MçGraw-Hill do Brasil, 1976.

13. SZARFARC, S.C. Prevalência de anemia nutricional entre gestantes matriculadas em Centros de Saúde do Estado de São Paulo. São Paulo, 1983. [Tese de LivreDocência - Faculdade de Saúde Pública da USP].
14. SZARFARC, S.C. et al. Avaliação da concentração de ferro em uma população de grávidas. Rev. Saúd públ., S. Paulo, 17: 200-7, 1983.

15. VITERI, F.E. et al. Normal hematological values in the Central American population. Brit. J. Haematol., 23: 189. 204. 1972.

Recebido para publicação em 12/03/1985

Reapresentado em 05/07/1985

Aprovado para publicação em 15/07/1985 